\title{
Purification of reduced upgraded titania slag by iron removal using mild acids
}

\author{
Jaehun Cho, Syamantak Roy, Amarchand Sathyapalan, Michael L. Free ${ }^{1}$, Z. Zak Fang, Weizhi \\ Zeng \\ Department of Metallurgical Engineering, University of Utah, Salt Lake City, Utah 84112
}

Keywords: titanium hydride, upgraded titania slag, iron removal, hydrochloric acid, boric acid

\begin{abstract}
In this study, we focused on removal of iron from upgraded titania slag (UGS) that has been reduced by hydrogen gas, magnesium powder and salts at $750^{\circ} \mathrm{C}$ for 5 hours. Reduced UGS is mainly composed of titanium hydride and oxide impurities that include iron, silicon, aluminum, and magnesium. Mild concentrations of hydrochloric and boric acids were used in this investigation to remove iron from reduced UGS. The concentration of hydrochloric and boric acids and the leaching temperature have been chosen as the prime variables to identify the optimum conditions and leaching characteristics for purification of reduced UGS. Both ambient pressure leaching and elevated pressure leaching were performed to assess the rate of iron removal from reduced UGS without titanium loss. It was observed that under the constraint that $1 \%(\mathrm{w} / \mathrm{w})$ of titanium hydride loss is the maximum amount tolerable, $0.1 \mathrm{M}$ hydrochloric acid at $140^{\circ} \mathrm{C}$ was found to be the most effective conditions for iron removal (87.63\%). A factorial design of experiment for equation modeling with three main factors (temperature, concentration of hydrochloric and boric acids) was performed and associated modeling results were in good

${ }^{1}$ Corresponding author: email: michael.free @utah.edu

(C) 2016. This manuscript version is made available under the Elsevier user license http://www.elsevier.com/open-access/userlicense/1.0/
\end{abstract}


agreement with experimental data. The overall rate of reaction seems to follow interfacial reaction controlled kinetics. The calculated activation energy of the reaction is $73.9 \mathrm{~kJ} / \mathrm{mol}$.

\section{Introduction}

Pure metallic titanium is of great industrial importance because of its use in various applications such as aviation (Zhou et al., 2005), aerospace (Boyer, 1996), biomedical (Niinomi, 2003), marine (Gorynin, 1999), and nuclear waste storage (Hua et al., 2005) due to its corrosion resistance, high specific strength, light weight, relatively high melting point, and chemical/heat stability. Moreover, it has great optical, mechanical, and electronic properties, as well as biocompatibility (Amarchand et al., 2000). Although it is predominantly found as rutile $\left(\mathrm{TiO}_{2}\right)$ and ilmenite $\left(\mathrm{FeTiO}_{3}\right)$, which are abundant in the earth's crust, the production cost is relatively high compared to competing metals such as steel and aluminum (Froes and Eylon, 1990). In general, titanium can be extracted from its ore by sulfate or chloride processes. Even though low grade ilmenite feed stock can be used, the major drawback of sulfate processes is the generation of a large quantity of waste iron sulfates. In the case of chloride processes, greenhouse gas is generated and it requires an upgraded starting material, which increases the cost of production (Middlemas et al., 2013).

The current commercial method for production of metallic titanium is the Kroll process in which metallic titanium is obtained by magnesiothermic reduction of titanium tetrachloride $\left(\mathrm{TiCl}_{4}\right)$ (Park et al., 2005), which is produced from the chlorination of titanium dioxide $\left(\mathrm{TiO}_{2}\right)$ or synthetic rutile. After that, additional leaching or heated vacuum distillation is carried out on the porous titanium sponge resulting from the reduction process as a further purification step (Kroll, 
1940). Although the Kroll process allows industry to produce high-purity metallic titanium with low oxygen level, there are several challenges. Firstly, developing a continuous reduction process is difficult due to the high vacuum, long-term processing, and the generation of titanium deposits on the inner wall of the reactor. Secondly, batch-type processing for long periods of time results in high labor power and low productivity (Takeda and Okabe, 2006). Furthermore, environmental issues such as generation of greenhouse gas and corrosive intermediate products like titanium tetrachloride are involved (Amarchand et al., 2000; Middlemas et al., 2013).

To mitigate the challenges of the Kroll process, several new alternative techniques with low cost and high purity and productivity have been suggested. Recently, titanium subchlorides such as titanium dichloride $\left(\mathrm{TiCl}_{2}\right)$ and titanium trichloride $\left(\mathrm{TiCl}_{3}\right)$ have been investigated as potential replacements for titanium tetrachloride $\left(\mathrm{TiCl}_{4}\right)$ feed. This process keeps the advantages of the Kroll process such as an oxygen-free environment and easy impurity-control and may increase the process speed (Takeda and Okabe, 2006). However, concerns such as handling volatile titanium tetrachloride require further technical and practical studies before industrial application. Another alternative technique is direct reduction from titanium dioxide by electrodeposition of titanium from ionic solutions, but this approach has several problems like difficulties in handling the redox cycle of multivalent titanium ions and reactive dendritic products (Chen et al., 2000). Recently, Fang et al. (2013) suggested titanium production from titanium hydride $\left(\mathrm{TiH}_{2}\right)$, which can be obtained by reduction of upgraded titania slag (UGS) or synthetic rutile under a controlled environment in the presence of magnesium and salts. After making titanium hydride, metallic titanium is produced by dehydrogenation of titanium hydride at about $500^{\circ} \mathrm{C}$ (Fang et al., 2013). In this new route, production costs and environmental problems can be significantly reduced and productivity can be improved compared to the Kroll process. The feed material, UGS is obtained 
by reduction of ilmenite ore in a blast furnace followed by roasting and acid leaching. However, one of the concerns in this process is the level of residual impurities such as iron oxide.

In this work, we are investigating the hydrometallurgical leaching characteristics using hydrochloric and boric acids associated with the removal of iron from UGS which has been reduced using hydrogen gas, magnesium powder and salts at $750^{\circ} \mathrm{C}$ for 5 hours (Fang et al., 2013). Reduced UGS is predominantly composed of titanium hydride and oxide impurities involving iron, silicon, aluminum, and/or magnesium. In fact, extraction of titanium using hydrochloric acid has been investigated extensively due to its low cost, reduced environmental issues, and potential for recycling compared to competing lixiviants such as sulfuric acid. However, most of the work has focused on extraction of titanium from ilmenite ore (El-Hazek et al., 2007; Ogasawara and Veloso de Araujo, 2000; Van Dyk et al., 2002). Thus, this study, the goal of which is iron removal from reduced UGS, can be differentiated from other research. The flow chart shown in Figure 1 illustrates the overall production of pure titanium from UGS. In this investigation, modest concentrations of hydrochloric and boric acids were used to reduce product loss. Boric acid was used to accelerate iron removal kinetics, because boric acid and borate tend to form complexes with hydrous iron oxides as shown in Equation 1 (Peak et al., 2003).

$$
\mathrm{Fe}^{3+}+\mathrm{H}_{3} \mathrm{BO}_{3}+\mathrm{H}_{2} \mathrm{O}=\mathrm{FeB}(\mathrm{OH})_{4}^{2+}+\mathrm{H}^{+}
$$

The stability constant of iron borate complexes is $1.0 \pm 0.2 \times 10^{-2} 25^{\circ} \mathrm{C}$ for an ionic strength of 0.68 (Elrod and Kester, 1980).

Important factors such as leaching temperature and concentrations were considered to identify the optimum condition and leaching characteristics for iron removal. 


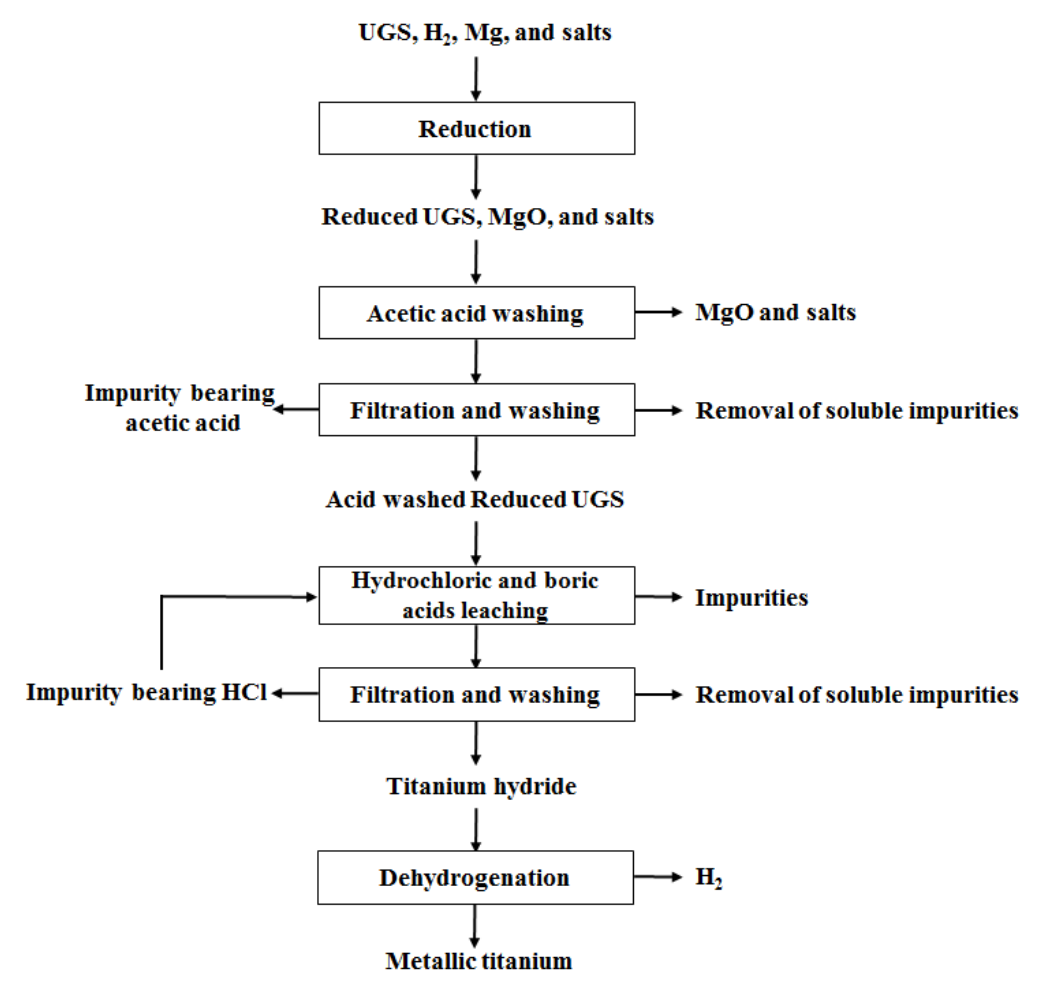

Figure 1. Flow sheet of new route for production of metallic titanium

\section{Materials \& methods}

\subsection{Preparation of reduced UGS by reduction and acetic acid leaching}

UGS is composed of about $96 \%$ titanium dioxide and $4 \%$ impurities. A mixture of UGS (less than $40 \mu \mathrm{m})$, magnesium powder and salts, such as magnesium chloride $\left(\mathrm{MgCl}_{2}\right)$ and potassium chloride $(\mathrm{KCl})$, with a ratio of $1: 1: 1$ by weight were placed in a furnace under hydrogen atmosphere and reduced for 5 hours at $750^{\circ} \mathrm{C}$. Excess magnesium and salts are used to help increase kinetics (Fang et al., 2013). The product resulting from the reduction is washed using 4.3 $\mathrm{M}$ acetic acid $\left(\mathrm{CH}_{3} \mathrm{COOH}\right)$ with a solid to liquid ratio of $1 \mathrm{~g}$ to $40 \mathrm{ml}$ for 2 hours at $70^{\circ} \mathrm{C}$ to remove magnesium compounds and salts from reduced UGS. After acetic acid washing and 
filtration, the residue was washed with deionized water and $0.05 \mathrm{M}$ hydrochloric acid. The sample so obtained is used as the feed in this study.

\subsection{Experimental procedures}

A solution of $200 \mathrm{ml}$ which contained 0.05 or $0.1 \mathrm{M}$ hydrochloric acids with or without $1 \mathrm{M}$ boric acid was prepared in a flask made of semi-clear polymethylpentene. A chemical and temperature resistant plug with a hole that allows for a thermometer insertion was applied on the top of the flask to maintain constant volume. The solution was mixed by a magnetic stirring bar at a rotational speed of 1,000 RPM and heated to the predetermined temperature, which is lower than the boiling point of water $\left(70,80\right.$, and $\left.90^{\circ} \mathrm{C}\right)$. Once the temperature reached the desired level, $2 \mathrm{~g}$ of reduced UGS obtained after acetic acid washing was added into the flask. The leaching experiment was run for 4 hours and samples were collected every hour for the study of reaction kinetics. In the cases where the experiments were conducted at higher temperature than the boiling point of water $\left(140\right.$ and $\left.190^{\circ} \mathrm{C}\right)$, a different experimental setup, which involved a pressure reactor, was used. A solution of $200 \mathrm{ml}$ which contained a low concentration of hydrochloric and boric acids with $2 \mathrm{~g}$ of reduced UGS after acetic acid washing was prepared in a polytetrafluoroethylene beaker and experiments were carried out in the pressure reactor. The desired temperature was achieved after 1 hour, and the experiments were carried out for 3 hours at the desired temperature. The pressure reactor was slowly cooled for 2 hours. After that, the solution was filtered and residue was washed with deionized water and $0.05 \mathrm{M}$ hydrochloric acid. The solution samples were analyzed by Inductively Coupled Plasma-Optical Emission Spectroscopy (ICP-OES). The solid samples were characterized using X-Ray Diffraction (XRD) 
and Scanning Electron Microscopy (SEM). The particle size distribution was determined using Laser Diffraction Particle Size Analyzer.

\section{Results and discussion}

\section{$\underline{\text { 3.1. Feed analysis }}$}

Table 1 shows the composition of reduced UGS immediately after reduction. Magnesium compounds and salts in Table 1 come from magnesium oxide, excess magnesium powder, magnesium chloride, and potassium chloride. The composition of the acetic acid washed reduced UGS is also listed in Table 1, showing the impurities which predominantly consist of iron and silicon oxides.

Table 1. Chemical composition of reduced UGS before and after acetic acid washing

\begin{tabular}{cccccc}
\hline Component (\%) & $\begin{array}{c}\text { Mg compounds } \\
\text { and salts }\end{array}$ & $\mathrm{Ti}$ & $\mathrm{Fe}$ & $\mathrm{Al}$ & $\mathrm{Si}$ \\
\hline Before washing & 79.79 & 19.52 & 0.22 & 0.07 & 0.41 \\
After washing & 0.36 & 96.20 & 1.07 & 0.35 & 2.02 \\
\hline
\end{tabular}

Figure 2 presents the mineralogical phases of the acetic acid washed, reduced UGS analyzed by XRD which shows that the crystalline phases of the products predominantly consist of titanium hydride and ferric oxide.

Figure 3 shows the surface morphology of reduced UGS after acetic acid washing as analyzed by SEM. This result can be used for comparison of surface morphology with that of the product after leaching in hydrochloric and boric acids. Although the sizes of individual particles seem 
very small, Figure 3 reveals larger agglomerates. The particle size analyzer supports this observation by reporting a range of particle size from 0.05 to $0.2 \mu \mathrm{m}$ as shown in Figure 4 .

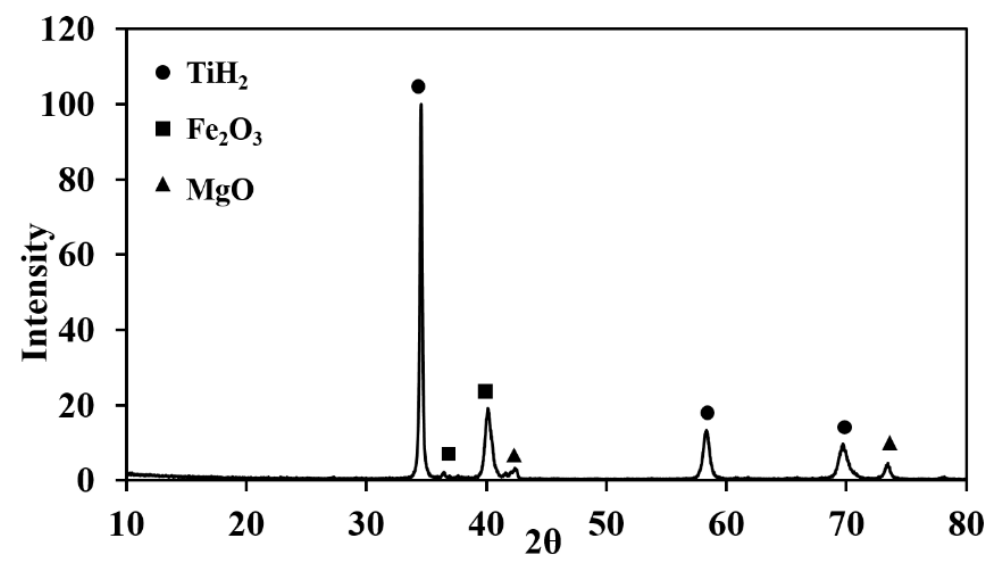

Figure 2. XRD pattern of the reduced UGS after acetic acid washing

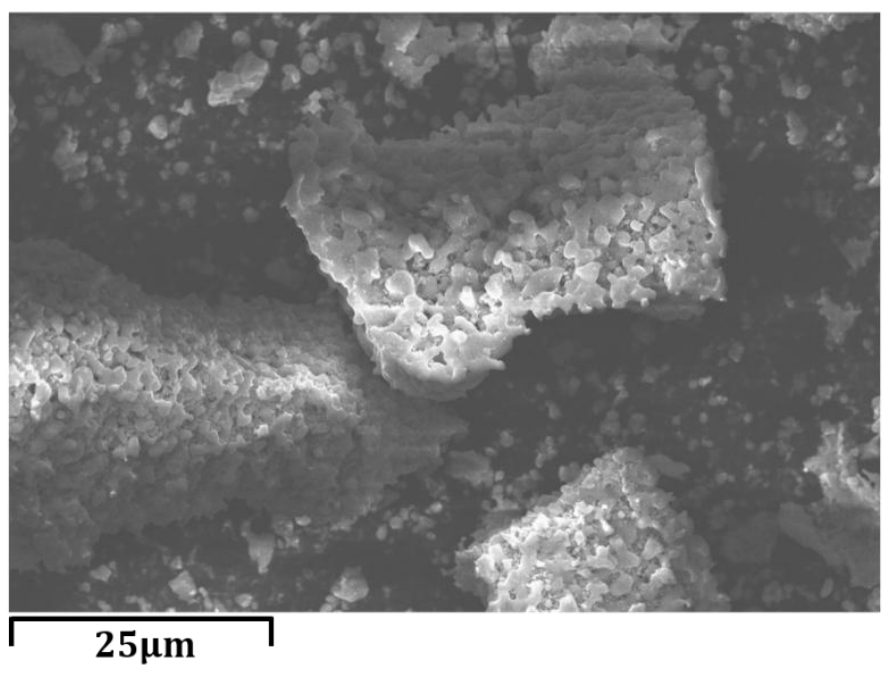

Figure 3. SEM image of reduced UGS after acetic acid washing 


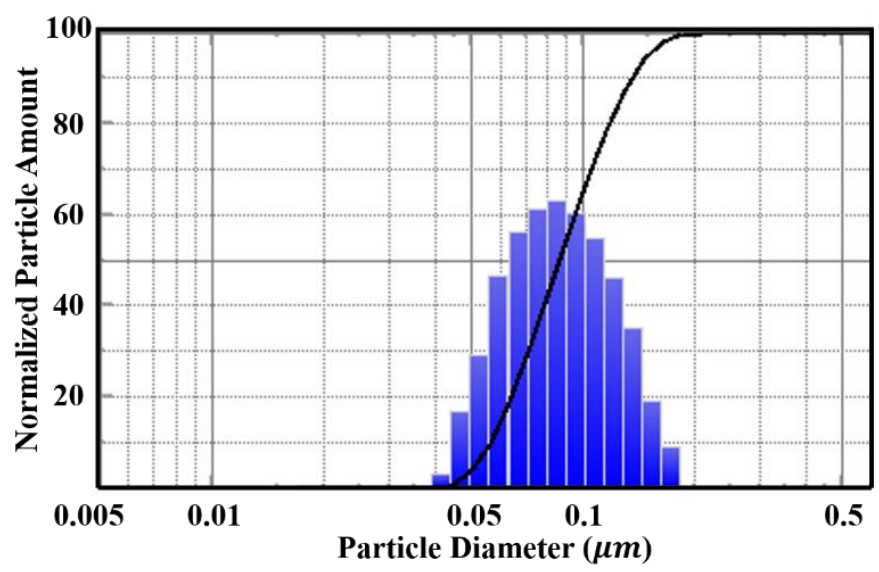

Figure 4. Particle size of reduced UGS after acetic acid washing

\subsection{Experimental results}

Table 2 shows the effect of each parameter on iron removal and titanium loss after 4 hours of leaching.

Table 2. Fe removal $\%$ and $\mathrm{Ti}$ loss $\%$ at different leaching parameters

\begin{tabular}{ccccc}
\hline Temp $\left({ }^{\circ} \mathrm{C}\right)$ & $\mathrm{HCl}(\mathrm{M})$ & Boric acid $(\mathrm{M})$ & Fe removal $(\%)$ & Ti loss $(\%)$ \\
\hline 90 & 0.05 & 0 & 27.30 & 0.07 \\
90 & 0.05 & 1 & 43.80 & 0.08 \\
90 & 0.1 & 0 & 55.01 & 0.50 \\
90 & 0.1 & 1 & 61.42 & 0.60 \\
140 & 0.05 & 0 & 79.59 & 0.06 \\
140 & 0.05 & 1 & 76.01 & 0.15 \\
140 & 0.1 & 0 & 87.63 & 0.21 \\
140 & 0.1 & 1 & 87.82 & 0.25 \\
190 & 0.05 & 0 & 79.19 & 0.22 \\
190 & 0.05 & 1 & 81.17 & 0.22 \\
190 & 0.1 & 0 & 88.26 & 0.12 \\
190 & 0.1 & 1 & 88.51 & 0.34 \\
\hline
\end{tabular}

* leaching time : 4 hours 


\subsubsection{Effect of hydrochloric acid}

One of the difficulties in the leaching of reduced UGS is that titanium hydride is easily dissolved in strong acid. In particular, titanium hydride tends to be converted to titanium trichloride in high concentrations of hydrochloric acid. The dissolution of titanium hydride can be expressed as [Astrelin et al., 2002]

$$
2 \mathrm{TiH}_{2}+6 \mathrm{H}^{+}+6 \mathrm{Cl}^{-}=2 \mathrm{TiCl}_{3}+5 \mathrm{H}_{2}
$$

Thus, before conducting the regular experiments with hydrochloric acid, the acceptable quantity of titanium hydride loss during the leaching process needs to be determined. Our research group determined arbitrarily that $1 \%(\mathrm{w} / \mathrm{w})$ of titanium loss is the maximum amount tolerable. Test results for titanium loss as a function of hydrochloric acid concentration are presented in Figure 5 under the leaching parameters in Table 3.

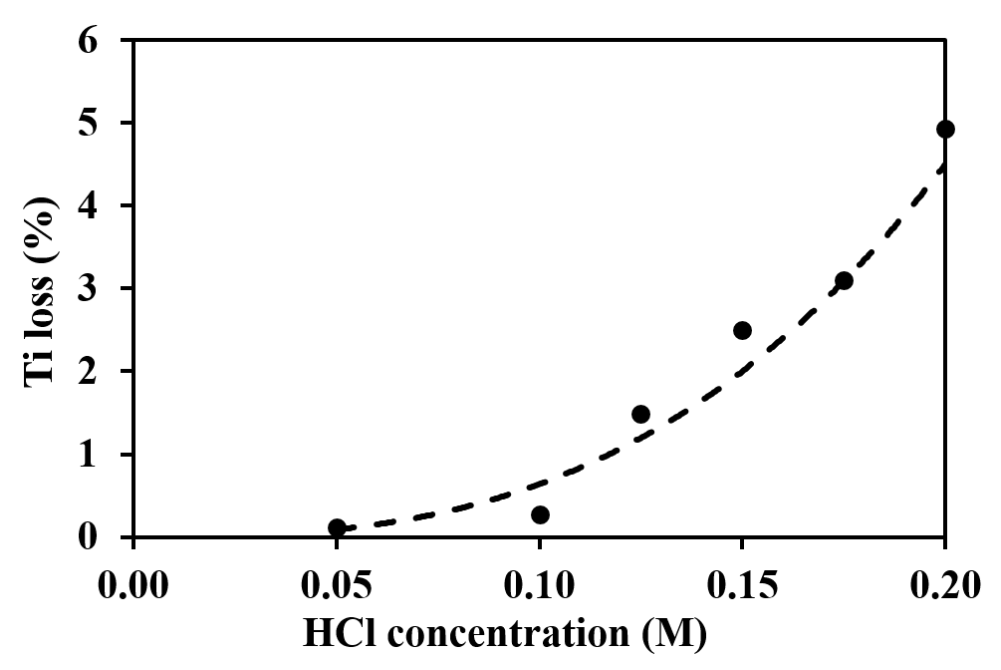

Figure 5. Titanium loss versus $\mathrm{HCl}$ concentration Table 3. Leaching parameters 


\begin{tabular}{ccccc}
\hline $\begin{array}{c}\text { Temperature } \\
\left({ }^{\circ} \mathrm{C}\right)\end{array}$ & $\begin{array}{c}\text { Time } \\
(\mathrm{h})\end{array}$ & $\begin{array}{c}\text { Stirring speed } \\
(\mathrm{RPM})\end{array}$ & $\begin{array}{c}\mathrm{S} / \mathrm{L} \\
(\mathrm{g}: \mathrm{ml})\end{array}$ & $\begin{array}{c}\text { Particle size } \\
(\boldsymbol{\mu m})\end{array}$ \\
\hline 90 & 4 & 1,000 & $1: 100$ & $0.05-0.2$ \\
\hline
\end{tabular}

Based on the results in Figure 5, the maximum concentration of hydrochloric acid that meets the requirement of less than $1 \%$ titanium loss is approximately $0.1 \mathrm{M}$. Thus, $0.05 \mathrm{M}$ and $0.1 \mathrm{M}$ hydrochloric acids were chosen to study the influence of hydrochloric acid concentration on the removal of iron as shown in Figure 6 under the leaching parameters in Table 3. The leaching with $0.1 \mathrm{M}$ hydrochloric acid shows $55.0 \%$ of iron removal after 4 hours along with $0.5 \%$ of titanium loss, whereas $0.05 \mathrm{M}$ hydrochloric acid removed only $27.3 \%$ of the iron. Much of the iron-removal taking place during leaching can be expressed generally as

$$
\mathrm{Fe}_{2} \mathrm{O}_{3}+6 \mathrm{H}^{+}+6 \mathrm{Cl}^{-}=2 \mathrm{FeCl}_{3}+3 \mathrm{H}_{2} \mathrm{O}
$$

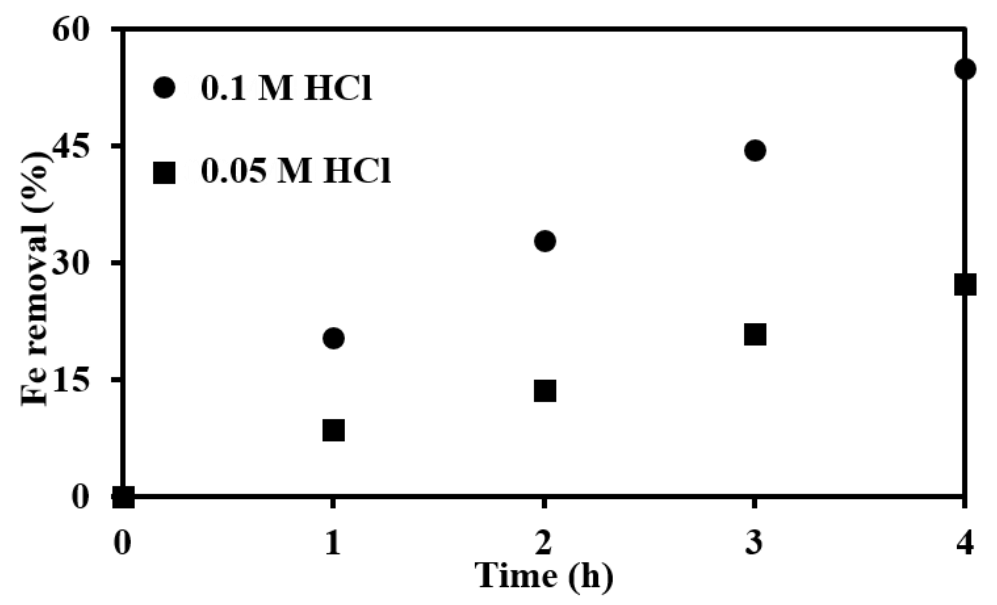

Figure 6. Comparison of iron removal and time for $0.05 \mathrm{M}$ and $0.1 \mathrm{M} \mathrm{HCl}$ at $90^{\circ} \mathrm{C}$

\subsubsection{Effect of boric acid}


Figure $7 \mathrm{a}$ shows the effect of boric acid in $0.05 \mathrm{M}$ hydrochloric acid. The iron removal efficiency significantly increased from $27.3 \%$ to $43.8 \%$ with the increase of boric acid. However, in $0.1 \mathrm{M}$ hydrochloric acid, the iron removal efficiency increased by only $6.4 \%$ when boric acid was added according to Figure $7 \mathrm{~b}$.

(a)

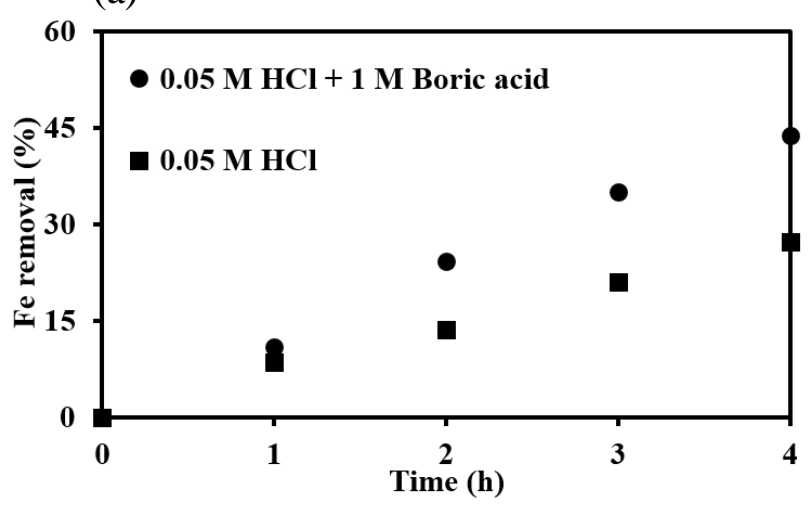

(b)

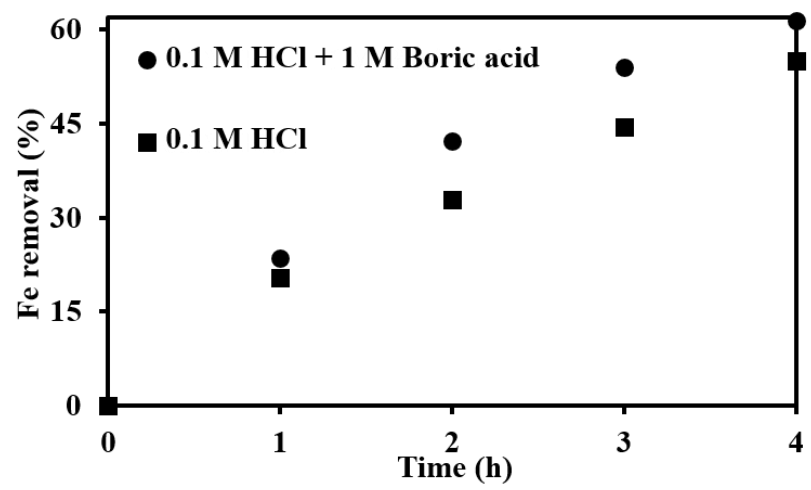

Figure 7. Comparison of iron removal versus time with/without boric acid at $90^{\circ} \mathrm{C}$

According to Peak et al. (2003), trigonal boric acid reacted with ferric iron would be converted to tetrahedral surface complexes as a result of the Lewis acidity of the boron metal center. Then hydrogen ions would be displaced from the tetrahedral boron, and ferric borate complexes are obtained as shown in Equation 1 (Elrod and Kester, 1980; Peak et al., 2003). However, this reaction is favorable in weak acid condition, which might explain why boric acid does not affect iron removal much at the higher concentration of hydrochloric acid (Figure 7).

\subsubsection{Effect of temperature}

The previous results show that the concentration of hydrochloric acid is restricted to $0.1 \mathrm{M}$ to prevent significant titanium loss, and boric acid does not help iron removal much in the presence of $0.1 \mathrm{M}$ hydrochloric acid in the solution. Thus, the effect of leaching temperature on iron 
removal was studied in the broad temperature range of $70-190^{\circ} \mathrm{C}$, with $0.1 \mathrm{M}$ hydrochloric acid and with/without $1 \mathrm{M}$ boric acid. Figure 8 shows that the iron removal $\%$ increases with the increase of temperature. It is noted that once the temperature reaches about $140^{\circ} \mathrm{C}$, no additional iron was removed. Thus, it appears that the limit of iron removal is near $90 \%$ if the titanium loss is kept below $1 \%$. It is also noted that iron removal without boric acid shows almost the same iron removal result as that with boric acid as shown in Figure 8. The result suggests high temperature and high acidity are not favorable for boric acid to form tetrahedral surface complexes with iron.

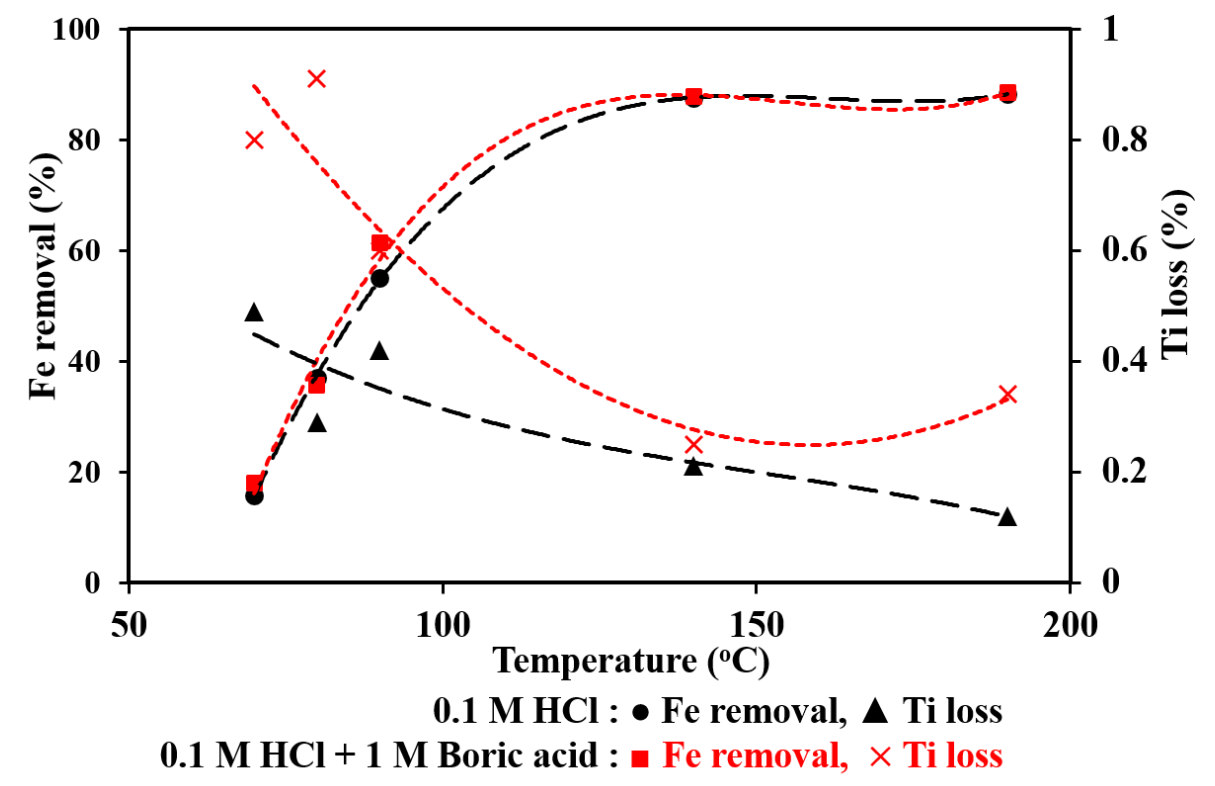

Figure 8. Fe removal and Ti loss versus temperature with $0.1 \mathrm{M} \mathrm{HCl}$ and with/without boric acid Therefore, the parameters that show the best results for iron removal based on the series of tests presented are $140^{\circ} \mathrm{C}, 0.1 \mathrm{M}$ hydrochloric acid and no boric acid. Weight percentage of iron removal under those optimized parameters is $87.63 \%$, which means that only $0.13 \%$ of iron remains in the leached, reduced UGS. The percentage of titanium was significantly changed from $96.2 \%$ to $99.45 \%$ after substantial impurity removal. According to the American Society 
for Testing and Materials (ASTM), the maximum tolerable amount of iron impurities in titanium sponge for general purpose is $0.15 \%$ by weight (ASTM B299-13, 2013). Thus, the reduced UGS that has been purified under the optimized parameters meets ASTM B299-13 iron specifications for general purposed titanium sponge. The whole composition of the reduced UGS leached by $0.1 \mathrm{M}$ hydrochloric acid with/without boric acid at $140^{\circ} \mathrm{C}$ is tabulated in Table 4 . The sample was analyzed by ICP-OES.

Table 4. Chemical composition of reduced UGS leached by $0.1 \mathrm{M} \mathrm{HCl}$ at $140^{\circ} \mathrm{C}$

\begin{tabular}{cccccc}
\hline Component $(\%)$ & $\mathrm{Mg}$ & $\mathrm{Ti}$ & $\mathrm{Fe}$ & $\mathrm{Al}$ & $\mathrm{Si}$ \\
\hline $0.1 \mathrm{M} \mathrm{HCl}$ & 0.05 & 99.45 & 0.13 & 0.12 & 0.25 \\
$0.1 \mathrm{M} \mathrm{HCl}+1 \mathrm{M} \mathrm{H}_{3} \mathrm{BO}_{3}$ & 0.07 & 99.13 & 0.13 & 0.10 & 0.57 \\
\hline
\end{tabular}

Figure 9 shows the XRD pattern of the reduced UGS obtained after 0.1 M hydrochloric acid leaching at $140^{\circ} \mathrm{C}$. According to Figure 9 , little titanium hydride was oxidized to titanium dioxide or metallic titanium, and most of impurities were below the detection limit for XRD.

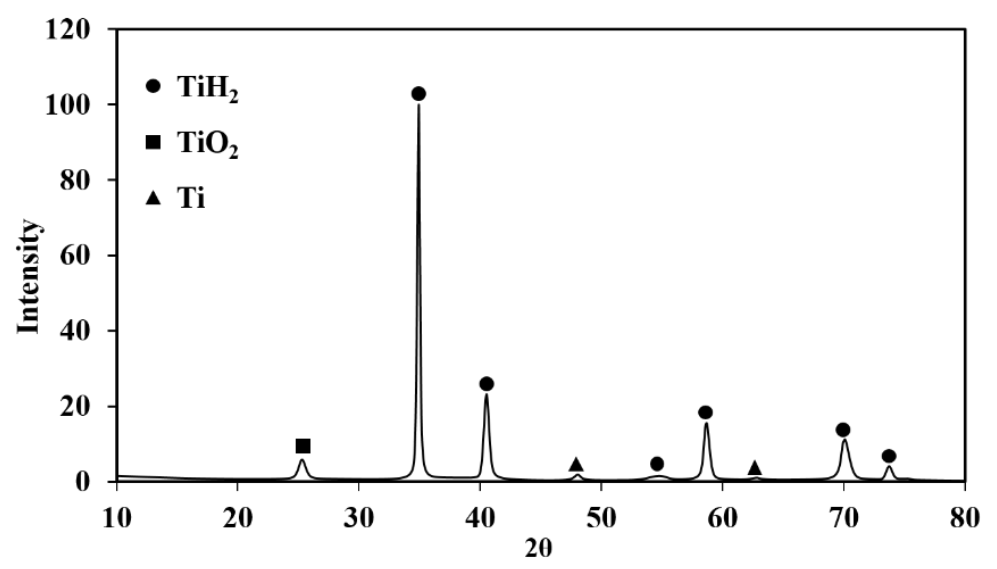

Figure 9. XRD pattern of the reduced UGS after $0.1 \mathrm{M} \mathrm{HCl}$ leaching at $140^{\circ} \mathrm{C}$ 


\subsection{Factorial design of experiments for equation modeling}

A model that can predict iron removal with the three parameters was established. Three levels of temperature $\left(90,140,190^{\circ} \mathrm{C}\right)$ and two levels of concentration of hydrochloric $(0.05,0.1 \mathrm{M})$ and boric acids $(0,1 \mathrm{M})$ were used as shown in Table 2 . The factorial design of experiments was analyzed by the statistical software 'Minitab'. The equation modeling for prediction of iron removal with three parameters is described in Equation 4.

$$
\begin{aligned}
& \text { Iron removal }(w t \%)=-164.116+2.56685 \mathrm{~T}+677.07[\mathrm{HCl}]+18.0998\left[\mathrm{H}_{3} \mathrm{BO}_{3}\right]- \\
& 0.00687205 \mathrm{~T}^{2}-2.89343 \mathrm{~T}[\mathrm{HCl}]-0.103388 \mathrm{~T}\left[\mathrm{H}_{3} \mathrm{BO}_{3}\right]
\end{aligned}
$$


where $\mathrm{T},[\mathrm{HCl}]$, and $\left[\mathrm{H}_{3} \mathrm{BO}_{3}\right]$ represent temperature in degrees Celsius, molarity of hydrochloric and boric acids, respectively and the $\mathrm{R}^{2}$ (adj.) value of the equation is 0.9541 . Also, in Figure 11, the residual versus order plot shows randomness and unpredictability where the sense of accuracy of the model is observed.

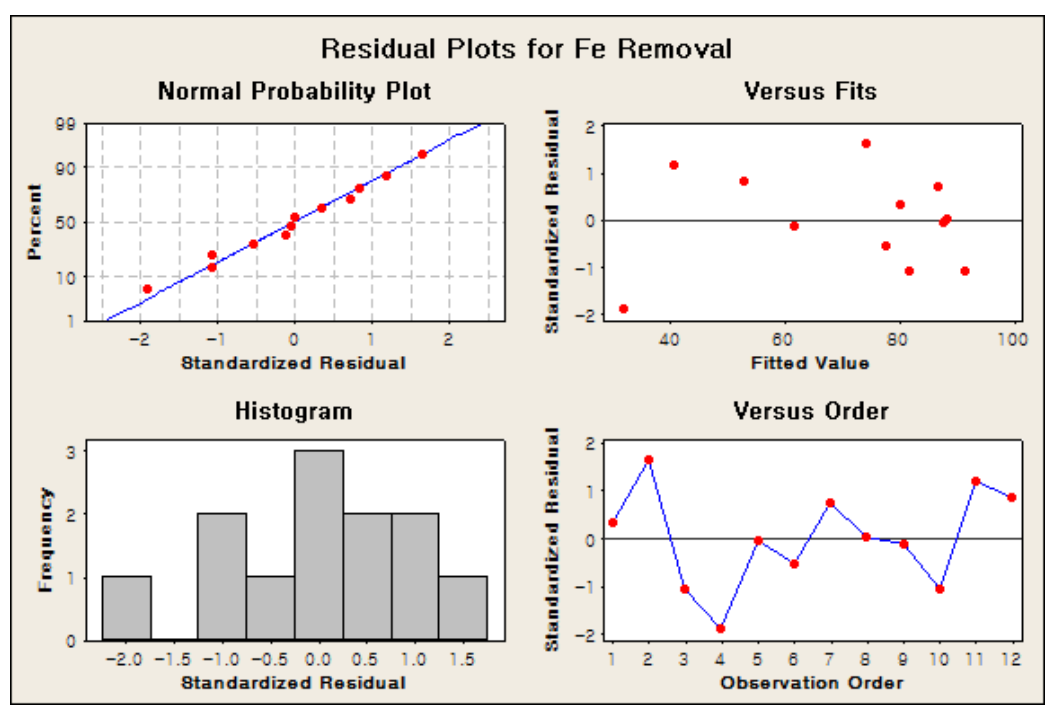

Figure 11. Residual plot for iron removal

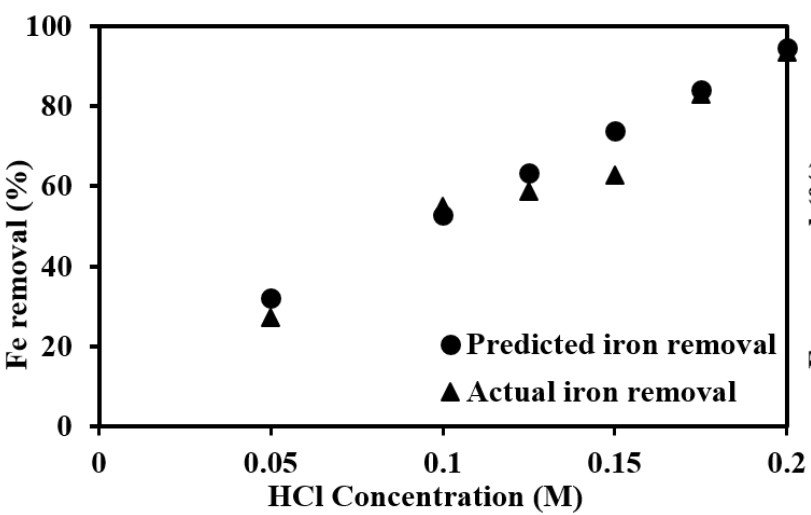

a) $\mathrm{Fe}$ removal versus $\mathrm{HCl}$ concentration at $90^{\circ} \mathrm{C}$

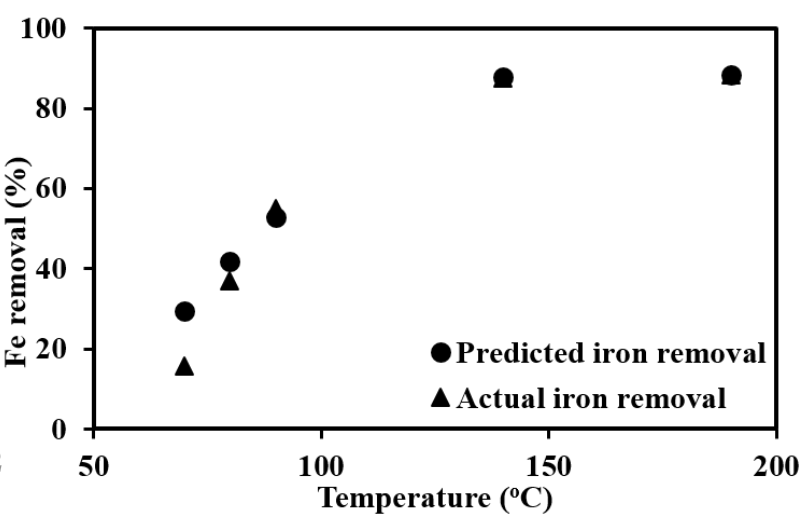

b) Fe removal versus Temperature in $0.1 \mathrm{M} \mathrm{HCl}$

Figure 12. Predicted and actual iron removal versus $\mathrm{HCl}$ concentration 
Figure 12a shows a comparison of iron removal at different concentrations of hydrochloric acid for predicted and actual results at $90^{\circ} \mathrm{C}$ and Figure $12 \mathrm{~b}$ presents a comparison of iron removal as a function of temperature with $0.1 \mathrm{M}$ hydrochloric acid. Overall, Figures $12 \mathrm{a}$ and $12 \mathrm{~b}$ show good agreement between predicted iron removal and actual iron removal. Note that although some of the data in Figure 12a show very high iron removal, concentrations above $0.1 \mathrm{M}$ hydrochloric acid result in significant titanium loss.

\subsection{Study of rate-controlling process}

The rate-controlling process and kinetic parameters were investigated on the basis of a reaction controlled kinetic model. If the overall rate of reaction is controlled by the interfacial reaction, a normalized time parameter, $\mathrm{t}^{*}$, can be compared to the fraction reacted, $\mathrm{X}$ as follows (Free, 2013; Szekely et al., 1976):

$$
t^{*}=1-(1-X)^{1 / 3}
$$

Experimental data obtained at three different temperatures, 70,80 , and $90^{\circ} \mathrm{C}$ were applied to Equation 5 to evaluate interfacial reaction controlled kinetics as illustrated by plotting $1-$ $(1-X)^{1 / 3}$ versus time as shown in Figure 13. 


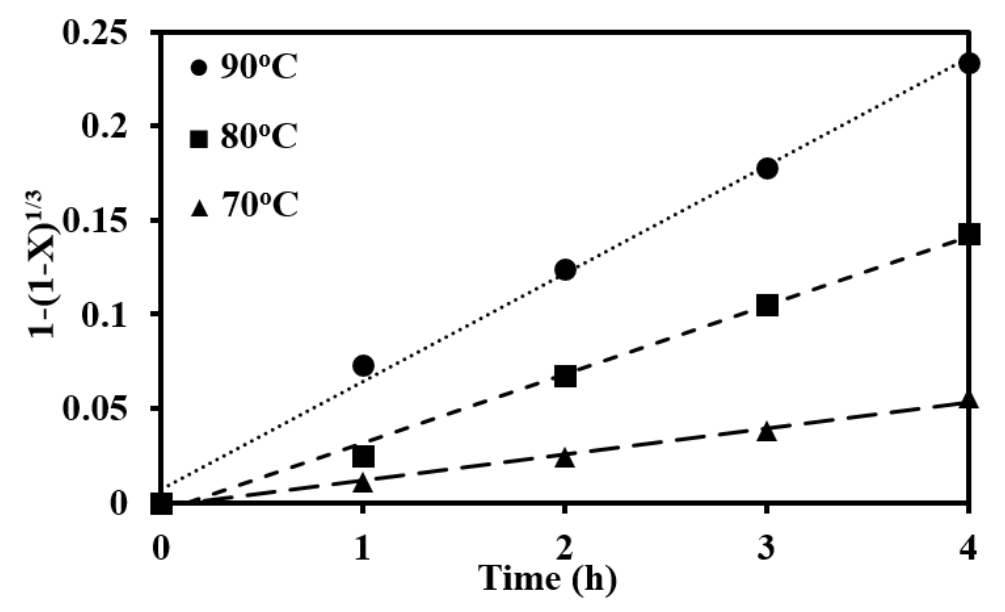

Figure 13. Relationship between the values of $\mathbf{1}-(\mathbf{1}-\mathbf{X})^{\mathbf{1 / 3}}$ and time at three temperatures

The value of $1-(1-X)^{1 / 3}$ is linearly related to leaching time, which suggests that the overall rate of reaction may be controlled by the interfacial reaction in the leaching process.

The activation energy can be used to evaluate the rate-controlling step. The Arrhenius equation, when rearranged for determining the activation energy is described in Equation 6 (Free, 2013).

$$
\ln k=\ln A-\frac{E_{a}}{R T}
$$

where $\mathrm{k}$ is the rate constant of a chemical reaction, $\mathrm{A}$ the pre-exponential factor, $\mathrm{E}_{\mathrm{a}}$ the activation energy, $\mathrm{R}$ the universal gas constant, and $\mathrm{T}$ the temperature in degrees Kelvin. Figure 14 shows the relationship between lnk and $10^{3} / \mathrm{T}$ in which the activation energy can be determined from the slope of the line. 


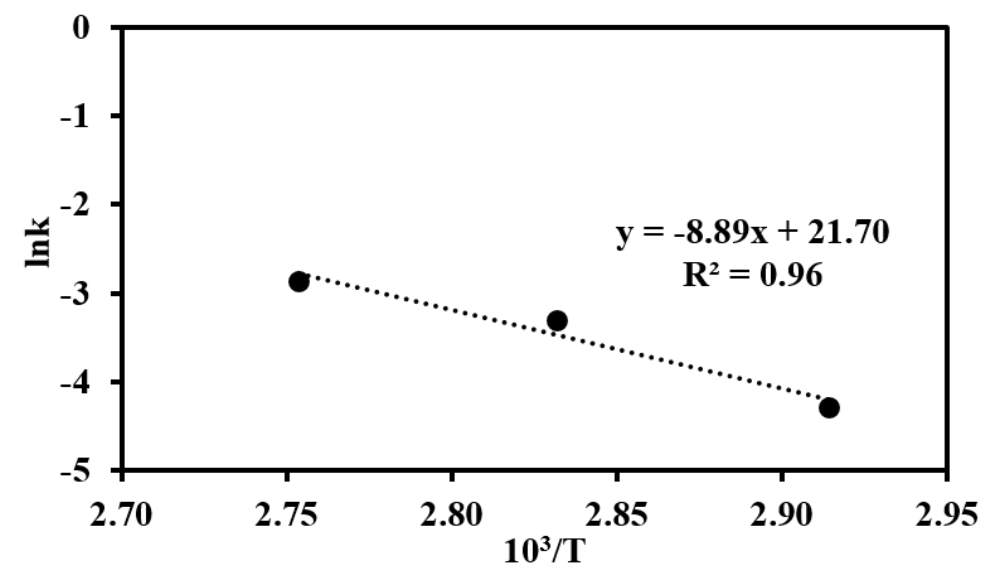

Figure 14. Arrhenius Plot

The activation energy was determined to be $73.9 \mathrm{~kJ} / \mathrm{mol}$, which suggests that interfacial reaction controlled kinetics can describe the observed data.

\subsection{Effect of mass transport}

Influence of mass transport on the reaction rate of leaching with $0.1 \mathrm{M}$ hydrochloric acid at $90^{\circ} \mathrm{C}$ was investigated by varying the stirring speed in the range of $100-1,000$ RPM.

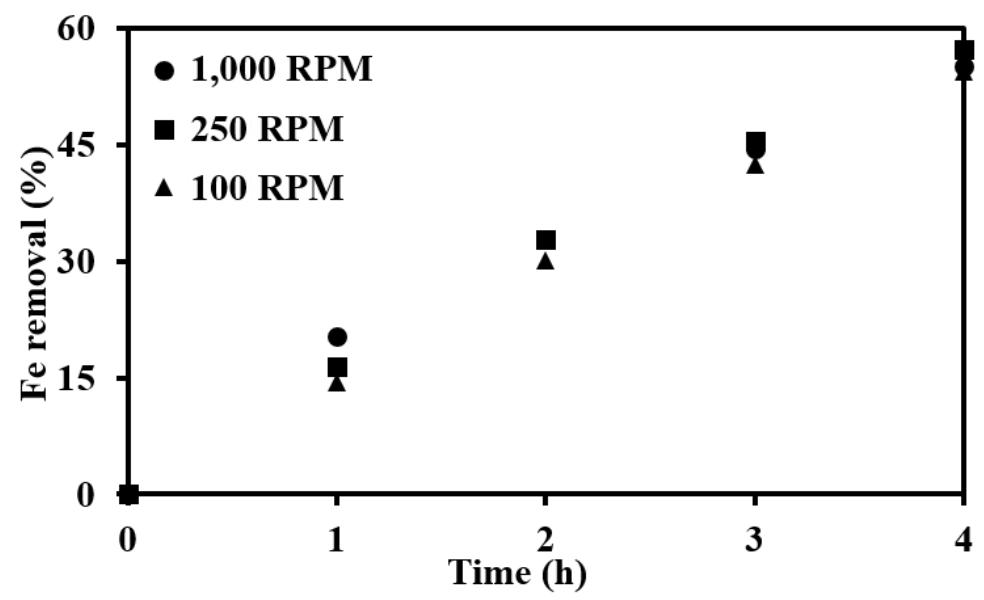

Figure 15. Iron removal versus time at three rotation speed 
The data in Figure 15 show that the reaction rate did not change significantly with increasing stirring speed, which implies that the overall rate of reaction is not controlled by mass transport through an outer boundary layer.

\section{Concluding remarks}

The leaching characteristics of iron removal from the reduced upgraded titania slag with mild hydrochloric and boric acids was studied under ambient pressure and elevated pressure.

(1) Under the constraint that $1 \%(\mathrm{w} / \mathrm{w})$ of titanium hydride loss is the maximum amount tolerable, $0.1 \mathrm{M}$ is the most effective concentration of hydrochloric acid for iron removal from the reduced UGS.

(2) Effect of boric acid on iron removal from reduced UGS was strongly dependent on acidity and temperature of the leaching environment. Solutions of $1 \mathrm{M}$ boric acid with $0.05 \mathrm{M}$ hydrochloric acid at $90^{\circ} \mathrm{C}$ showed significant improvement of iron removal, but with $0.1 \mathrm{M}$ hydrochloric acid, a reduced effect was observed. At higher temperature $\left(140,190^{\circ} \mathrm{C}\right)$, boric acid did not affect iron removal, regardless of the concentration of hydrochloric acid.

(3) Extent of iron removal increased significantly with the increase of temperature. Once temperature reaches about $140^{\circ} \mathrm{C}, 87.63 \%$ of iron removal was achieved with $0.1 \mathrm{M}$ hydrochloric acid. Additional iron removal was not observed at higher temperature $\left(190^{\circ} \mathrm{C}\right)$.

(4) A factorial design of experiment (FDE) for equation modeling was carried out to study the influence of three factors (temperature and concentrations of hydrochloric and boric acids) on 
iron removal. The predicted iron removal obtained by the FDE model showed good agreement with actual iron removal within the range of condition evaluated.

(5) The overall rate of reaction seems to follow interfacial reaction controlled kinetics. The activation energy of the reaction was determined to be $73.9 \mathrm{~kJ} / \mathrm{mol}$.

(6) Changes in mass transport through stirring speed changes do not affect the reaction rate significantly.

\section{Acknowledgments}

The authors are thankful for financial support of the Direct Reduction of Titanium Slag (DRTS) project (Grant \# 55800707) by ARPA-E of the US Department of Energy.

\section{Reference}

Amarchand, S., Rama Mohan, T.R., Ramakrishnan, P., 2000. A novel chemical solution technique for the preparation of nano size titanium powders from titanium dioxide, Advanced Powder Technol., 11 (4), 415-422.

ASTM B299-13, 2013. Standard specification for titanium sponge, ASTM International, West Conshohocken, PA.

Astrelin, I.M., Prokofyeva, G.N., Suprunchuk, V.I., Knyazev, Y.V., Panashenko, V.M., Morozov, I.A., Morozova, R.A., 2001. Interaction of $\mathrm{TiH}_{\mathrm{x}}(\mathrm{x}>2)$ with solutions of some acids and alkalies, in: Veziroglu, T.N., Zaginaichenko, S.Y., Schur, D.V., Trefilov, V.I. (Eds.), Hydrogen Materials Science and Chemistry of Metal Hydrides, Kluwer Academic Publishers, Netherlands, pp. 133-140. 
Boyer, R.R., 1996. An overview on the use of titanium in the aerospace industry, Materials Science and Engineering: A, 213 (1-2), 103-114.

Chen, G.Z., Fray, D.J., Farthing, T.W., 2000. Direct electrochemical reduction of titanium dioxide to titanium in molten calcium chloride, Nature, 407, 361-364.

El-Hazek, N., Lasheen, T.A., El-Sheikh, R., Zaki, S.A., 2007. Hydrometallurgical criteria for $\mathrm{TiO}_{2}$ leaching from Rosetta ilmenite by hydrochloric acid, Hydrometallurgy, 87, 45-50.

Elrod, J.A., Kester, D.R., 1980. Stability constants of iron (III) borate complexes, Journal of Solution Chemistry, 9 (11), 885-894.

Fang, Z.Z., Middlemas, S., Fan, P., Guo, J., 2013. A novel chemical pathway for producing low cost Ti by direct reduction of Ti-slag, Journal of American Chemical Society, 135 (49), 1824818251.

Free, M.L., 2013. Hydrometallurgy: Fundamentals and Applications, John Wiley \& Sons, lnc., Hoboken, New Jersey.

Froes, F. H., Eylon, D., 1990. Powder metallurgy of titanium alloys, Int. Mater. Rev. 35 (3), $162-$ 182.

Gorynin, I.V., 1999. Titanium alloy for marine application, Materials Science and Engineering: A, 263 (2), 112-116.

Hua, F., Mon, K., Pasupathi, P., Gordon, G., Shoesmith, D., 2005. A review of corrosion of titanium grade 7 and other titanium alloys in nuclear waste repository environments, Corrosion, $61(10), 987-1003$.

Kroll, W. J., 1940. The production of ductile titanium, Transactions of the Electrochemical Society, 78, 35-47. 
Middlemas, S., Fang, Z.Z., Fan, P., 2013. A new method for production of titanium dioxide pigment, Hydrometallurgy, 131-132, 107-113.

Niinomi, M., 2003. Recent research and development in titanium alloys for biomedical applications and healthcare goods, 4 (5), 445-454.

Ogasawara, T., Veloso de Araujo, R.V., 2000. Hydrochloric acid leaching of a pre-reduced Brazilian ilmenite concentrate in an autoclave, Hydrometallurgy, 56, 203-216.

Park, I., Abiko, T., Okabe, T.H., 2005. Production of titanium powder directly from $\mathrm{TiO}_{2}$ in $\mathrm{CaCl}_{2}$ through an electronically mediated reaction (EMR), Journal of Physics and Chemistry of Solid, 66, 410-413.

Peak, D., Luther III, G.W, Sparks, D.L, 2013. ATR-FTIR spectroscopic studies of boric acid adsorption on hydrous ferric oxide, Geochimica et Cosmochimica Acta, 67 (14), 2551-2560.

Szekely, J., Evans, J.W., Sohn, H.Y., 1976. Gas-Solid Reaction, Academic Press, New York.

Takeda, O., Okabe, T.H., 2006. High-speed titanium production by magnesiothermic reduction of titanium trichloride, Materials Transactions, 47 (4), 1145-1154.

Van Dyk, J.P., Vegter, N.M., Pistorius, P.C., 2002. Kinetics of ilmenite dissolution in hydrochloric acid, Hydrometallurgy, 65, 31-36.

Zhou, Y.G., Zeng, W.D., Yu, H.Q., 2005. An investigation of a new near-beta forging process for titanium alloys and its application in aviation components, Materials Science and Engineering: A, 393 (1-2), 204-212. 